\title{
Fetal growth is increased by maternal type 1 diabetes and HLA DR4-related gene interactions
}

\author{
M. Hummel • S. Marienfeld • M. Huppmann • \\ A. Knopff • M. Voigt • E. Bonifacio • A.-G. Ziegler
}

Received: 13 April 2006 / Accepted: 7 January 2007 / Published online: 20 February 2007

(C) Springer-Verlag 2007

\begin{abstract}
Aims/hypothesis Intrauterine growth in non-diabetic pregnancies is reported to be influenced by type 1 diabetes susceptibility genes. In particular, the high-risk HLA DR4_DQB1*0302 haplotype is associated with increased birthweight. The aim of this study was to determine whether HLA DR4 was associated with increased birthweight in a maternal diabetes environment and whether effects persisted during early childhood.

Subjects and methods Birthweight and gestational age were obtained in singleton births from mothers with type 1 diabetes $(n=1161)$ or whose fathers or siblings have type 1 diabetes $(n=872)$. Weight and height at ages 2 and 5 years were obtained from paediatric records. Data were adjusted for (gestational) age and sex and expressed as percentiles of German reference data. HLA DR typing was obtained for all children and 1090 children also had insulin gene (INS) variable number of tandem repeats (VNTR) typing.

Results Maternal type 1 diabetes was associated with increased birthweight, gestational age and birthweight
\end{abstract}

Electronic supplementary material The online version of this article (doi:10.1007/s00125-007-0607-2) contains supplementary material, which is available to authorised users.

\footnotetext{
M. Hummel · S. Marienfeld · M. Huppmann · A. Knopff •

A.-G. Ziegler $(\bowtie)$

Institute of Diabetes Research and Academic Hospital Schwabing,

Kölner Platz 1,

80804 München, Germany

e-mail: anziegler@1rz.uni-muenchen.de

M. Voigt

Paediatric Department, EMA-University Greifswald,

Greifswald, Germany

E. Bonifacio

San Raffaele Scientific Institute,

Milan, Italy
}

percentiles (all $p<0.0001$ ). In children of mothers with type 1 diabetes, birthweight percentile was further related to maternal $\mathrm{HbA}_{1 \mathrm{c}}$ during pregnancy $(r=0.26 ; p<0.0001)$ and was independently increased if children had HLA DR4 alleles (76th vs 64th percentile; $p<0.0001$ ). HLA DR4 was not associated with birthweight in children of non-diabetic mothers. Birthweight was not associated with INS VNTR genotypes. High birthweight, but not HLA DR4 was associated with increased weight and BMI at ages 2 and 5 years $(p<0.0001)$.

Conclusions/interpretation Our findings are consistent with the hypothesis that a diabetic intrauterine environment interacts with gene(s) marked by the type 1 diabetes susceptibility HLA DR4 alleles to increase fetal growth.

Keywords Birthweight - Gestation · Gestational age · Growth rate $\cdot \mathrm{HbA}_{1 \mathrm{c}} \cdot \mathrm{HLA} \cdot I N S \mathrm{VNTR} \cdot$ Intrauterine $\cdot$ Type 1 diabetes

Abbreviation
VNTR variable number of tandem repeats

\section{Introduction}

There is substantial interest in increasing weight trends during childhood and in the relevance of childhood weight to health and disease. In Western countries, the proportion of overweight children has reached unprecedented levels and rising weight trends are likely to contribute to increased incidences of type 1 diabetes, type 2 diabetes and cardiovascular disease $[1,2]$.

Weight during childhood is largely attributed to postnatal diet and exercise habits [3,4]. Birthweight has also 
been reported to affect weight trends in children and adolescents, suggesting that some programming of weight status occurs in utero [5-8]. Factors that affect birthweight within demographically similar births include pre-pregnancy weight, weight gain during pregnancy, maternal smoking, parity and disease [9]. Maternal diabetes increases birthweight $[10,11]$. More recently, genes that influence the risk of type 1 diabetes have also been shown to affect birthweight and/or childhood weight gain [12, 13]. In particular, the HLA DR4_DQB1*0302 haplotype, which is strongly associated with type 1 diabetes, was found to be associated with increased birthweight in the Swedish population [12]. Such relationships have fuelled interest and debate on a possible central role of nutrition and childhood weight status in the pathogenesis of type 1 and type 2 diabetes [14, 15].

Since 1989, we have prospectively followed from birth children of parents or siblings with type 1 diabetes $[16,17]$. In these children anthropometric data such as weight and height are obtained throughout childhood. More than half of the children are exposed to a diabetic intrauterine environment, and most of the children inherit genes from at least one parent with type 1 diabetes. Thus the cohort has a relatively high frequency of HLA DR4 and is useful to test the hypothesis that the HLA DR4_DQB1*0302 haplotype influences birthweight. The aim of this study was to determine whether HLA DR4 status increased birthweight in a maternal diabetes environment and whether the effects persisted during early childhood.

\section{Subjects and methods}

Subjects Children followed prospectively from birth in the German BABYDIAB [16-18] and BABYDIET [19] studies were included. Recruitment into the studies began in 1989 (BABYDIAB) and 2000 (BABYDIET). A total of 2,399 children have been screened for participation in these studies. Birthweight and gestational age at birth and HLA DR typing were obtained for 2,033 singleton births (1,325 from BABYDIAB, 708 from BABYDIET) from 1,728 families (in 279 families more than one child participated in the studies). These 2,033 offspring included 1,161 newborns of mothers with type 1 diabetes and 872 newborns whose mothers were healthy, but whose fathers $(n=819)$ or siblings $(n=53)$ had type 1 diabetes. Participants of non-western ethnic groups comprised $1 \%$ of the study cohort and included $0.8 \%$ of Turkish descent. All families gave written informed consent to participate in the study. The proportion of children who have dropped out of the studies was $20 \%$ by age 5 years. The studies were approved by the ethics committee of Bavaria, Germany and the ethics committee of the Ludwig-Maximilians Universi- ty, respectively (Bayerische Landesärztekammer no. 95357 and Ethikkommission der Medizinischen Fakultät der Ludwig-Maximilians Universität no. 329/00).

Birthweight percentiles and weight, height and BMI percentiles at follow-up Perinatal and anthropometric data were collected from each child's paediatric record at birth, at ages 9 months and 2 years, and every 3 years thereafter. Paediatric records were completed by trained staff at delivery and by paediatricians at clinical visits after birth. Gestational age was determined on the basis of the last menstrual period and expressed as weeks. Weight and height were determined at paediatric visits. Birthweight was adjusted for sex and gestational age, and data on height, weight and BMI at follow-up were adjusted for sex and the exact age at examination; all four of these characteristics were expressed as a percentile of the reference German population as determined by the German Perinatal Registry [20]. This registry contains data from 563,480 singleton births in 1992 (complete data from 14 of the 16 German federal states). Validation of the 1992 data was made from a second cohort of 1,800,000 offspring born between 1995 and 1997 (M. Voigt, unpublished data). Data expressed as percentiles will have a similar distribution to those in the reference population, if their median is at the 50th percentile and their interquartile range is the 25 th to the 75 th percentile.

HLA and insulin gene variable number of tandem repeats genotyping HLA DR and DQ genotypes were determined in children and in 1,275 parents. HLA-DRB1, HLA-DQA1 and $H L A-D Q B 1$ alleles were typed using PCR-amplified DNA and non-radioactive sequence-specific oligonucleotide probes as described previously [21, 22].

Insulin gene (INS) variable number of tandem repeats (VNTR) typing was performed in 1,090 children from the BABYDIAB study only. It was determined by $H p h \mathrm{I}$ digestion of PCR amplification products of the region of interest as described previously [23]. The single nucleotide polymorphism identified by this method is in almost complete linkage disequilibrium with the INS VNTR [23].

Maternal data The age of the mother at delivery was obtained from the child's paediatric record. The age of onset of maternal type 1 diabetes was reported by the mother in a questionnaire given to the mothers before or at delivery. Mothers with type 1 diabetes also reported whether they had overt nephropathy, which was defined as macroalbuminuria. $\mathrm{HbA}_{1 \mathrm{c}}$ from the last trimester of pregnancy was also reported by mothers with type 1 diabetes in this questionnaire. $\mathrm{HbA}_{1 \mathrm{c}}$ was determined as part of clinical care of the patients. $\mathrm{HbA}_{1 \mathrm{c}}$ was measured locally by undisclosed methods. $\mathrm{HbA}_{1 \mathrm{c}}$ values were provided by 606 of 1,161 mothers with type 1 diabetes. 
Statistical analysis Quantitative variables were analysed as continuous variables unless otherwise stated. Birthweight percentiles were also expressed as categories representing tertiles of the normative population. Maternal $\mathrm{HbA}_{1 \mathrm{c}}$ was also expressed as categories representing normal $(<5.6 \%)$, moderately elevated $(5.6-6.5 \%)$ and highly elevated $(>6.5 \%)$ values. HLA DR4 status was categorised as HLA DR4-positive if the child had at least one DR4 allele and HLA DR4-negative if the child had no DR4 alleles. HLA DR4 status was also categorised as: (1) DR4-negative, DR4-heterozygous and DR4-homozygous; and (2) no HLA DR4 allele, HLA DR4 from the mother, HLA DR4 from the father, HLA DR4 from both parents, and parental origin of HLA DR4 unknown. Parity status was categorised as first child, second child or subsequent births. Comparisons of birthweight and gestational ages between groups were performed using the Student's $t$ test. Comparisons of birthweight percentile between groups was performed using the Mann-Whitney $U$ test. Correlations between continuous data (birthweight, gestational age, birthweight percentiles, $\mathrm{HbA}_{1 c}$, age of mother at child's birth, diabetes duration at child's birth, age of mother at diabetes onset) were performed using linear regression.

Multivariate analysis was performed for associations with birthweight using linear regression since birthweight was normally distributed. Factors and covariates included in the multivariate analysis were: (1) in the total cohort: gestational age, sex of the child, DR4 status of the child (positive or negative), maternal diabetes, maternal age and parity status; and (2) within children of mothers with type 1 diabetes: gestational age, sex of the child, parity status, DR4 status of the child (positive or negative), maternal $\mathrm{HbA}_{1 c}$ during pregnancy, maternal age, maternal diabetes duration and nephropathy including macroalbuminuria. The interaction between maternal diabetes and child HLA DR4 status on birthweight was tested in the general linear model by including maternal diabetes, HLA DR4 status and maternal diabetes $\times$ HLA DR4 status in a separate model.

For childhood growth analyses, 66 children who developed multiple islet autoantibodies or diabetes before age 5 years were excluded, since growth rates have been reported to differ in children who develop diabetes. Relationships between age- and sex-corrected weight percentile or BMI percentile and birthweight, gestational age, HLA DR4 status of the child (DR4-positive or DR4negative) and maternal diabetes were analysed using the general linear model for repeated measures $(2$ years and 5 years). For all analyses, a two tailed $p$ value of 0.05 was considered significant. All statistical analyses were performed using the Statistical Package for Social Science (SPSS 14.0; SPSS, Chicago, IL, USA).

\section{Results}

Maternal diabetes affects intrauterine growth In offspring of mothers with type 1 diabetes birthweight was higher and gestational age was lower than in offspring of healthy mothers (Table 1). Birthweight percentile (adjusted for sex and gestational age) in children of mothers without diabetes (fathers or siblings with type 1 diabetes) was similar to the reference population data (median: 48th percentile) (Table 2). In comparison, the median birthweight percentile in children of mothers with type 1 diabetes was at the 70th percentile $(p<0.0001)$.

Parity affected birthweight and birthweight percentile, but did not affect gestational age (Table 2). In children born to mothers with type 1 diabetes, birthweight, gestational age and birthweight percentile were lower when mothers had overt nephropathy $(p<0.0001)$. Neither birthweight nor birthweight percentile were correlated with the age of the
Table 1 Characteristics of the cohort

${ }^{\mathrm{a}} p<0.0001$ vs children of nondiabetic mothers.

\begin{tabular}{lll}
\hline & $\begin{array}{l}\text { Non-diabetic mothers } \\
n=872\end{array}$ & $\begin{array}{l}\text { Type } 1 \text { diabetes mothers } \\
n=1,161\end{array}$ \\
\hline Pregnancy $\mathrm{HbA}_{1 c}(\%$; mean $[\mathrm{SD}])$ & Not determined & $5.8(0.9)$ \\
Mother's age (years; mean [SD]) & $31.1(4.5)$ & $30.4(4.4)^{\mathrm{a}}$ \\
Diabetes duration in mother (years; mean [SD]) & Not applicable & $13.4(7.8)$ \\
Nephropathy with macroalbuminuria $(n$ [\%]) & Not determined & $33(3)$ \\
Gestation (weeks; mean [SD]) & $39.4(1.7)$ & $38.3(1.8)^{\mathrm{a}}$ \\
Sex of child (male/female) & $461 / 411$ & $601 / 560$ \\
First born children $(n[\%])$ & $471(54)$ & $676(58)$ \\
Birthweight $(\mathrm{g} ;$ mean $[\mathrm{SD}])$ & $3,424(527)$ & $3,504(624)^{\mathrm{a}}$ \\
DR4 in child $(n[\%])$ & $431(49)$ & $542(47)$ \\
$I N S$ VNTR in child $(n[\%])$ & & $439(63.7)$ \\
Class I/I & $252(62.8)$ & $225(32.7)$ \\
Class I/III & $129(32.2)$ & $25(3.6)$ \\
Class III/III & $20(5)$ & \\
\hline
\end{tabular}


Table 2 Birthweight (median percentile adjusted for gestational age and sex [interquartile range]) in relation to maternal diabetes status and child parity, $\mathrm{HbA}_{1 c}$, HLA and INS VNTR status

${ }^{a} p<0.0001$ vs first offspring;

${ }^{\mathrm{b}} p<0.05$ vs first offspring;

${ }^{c} p<0.0001$ vs children born to mothers with a pregnancy $\mathrm{HbA}_{1 \mathrm{c}}<5.6 \%$; ${ }^{\mathrm{d}} p=0.003 \mathrm{vs}$ children born to mothers with a pregnancy $\mathrm{HbA}_{1 \mathrm{c}}<5.6 \%$; ${ }^{\mathrm{e}} p<0.0001$ vs children born to mothers without nephropathy; f $p<0.0001$ vs DR4-negative children of mothers with type 1 diabetes

\begin{tabular}{|c|c|c|c|}
\hline & $n(\%)$ & Non-diabetic mother & Type 1 diabetes mother \\
\hline Total & 2,033 & $48(25-72)$ & $70(37-91)$ \\
\hline \multicolumn{4}{|l|}{ Parity } \\
\hline First offspring & $1,147(56)$ & $44(21-67)$ & $63(32-87)$ \\
\hline Second offspring & $713(35)$ & $55(30-76)^{\mathrm{a}}$ & $78(49-96)^{\mathrm{a}}$ \\
\hline Subsequent offspring & $173(9)$ & $60(33-84)^{\mathrm{a}}$ & $85(43-95)^{\mathrm{b}}$ \\
\hline \multicolumn{4}{|l|}{ Pregnancy $\mathrm{HbA}_{1 c}$} \\
\hline$<5.6 \%$ & $247(21)$ & - & $55(27-84)$ \\
\hline $5.6-6.5 \%$ & $267(24)$ & - & $75(48-91)^{\mathrm{c}}$ \\
\hline$>6.5 \%$ & $92(4)$ & - & $85(60-96)^{\mathrm{c}}$ \\
\hline Missing cases & $555(48)$ & - & $69(32-93)^{\mathrm{d}}$ \\
\hline \multicolumn{4}{|l|}{ Overt nephropathy } \\
\hline No & $1,067(91)$ & - & $72(39-92)$ \\
\hline Yes & $33(3)$ & - & $37(13-72)^{\mathrm{e}}$ \\
\hline Missing cases & $69(6)$ & - & $64(24-91)$ \\
\hline \multicolumn{4}{|l|}{ DR4 status of child } \\
\hline DR4-negative & $1,060(52)$ & $49(26-74)$ & $64(30-90)$ \\
\hline DR4-heterozygous & $868(43)$ & $50(24-70)$ & $75(43-93)^{f}$ \\
\hline DR4-homozygous & $105(5)$ & $40(21-63)$ & $83(53-93)^{\mathrm{f}}$ \\
\hline \multicolumn{4}{|l|}{ INS VNTR of child } \\
\hline Class I/I & $691(34)$ & $49(21-72)$ & $68(34-92)$ \\
\hline Class I/III & $354(18)$ & $51(26-80)$ & $68(35-91)$ \\
\hline Class III/III & $45(2)$ & $41(13-69)$ & $78(43-89)$ \\
\hline Missing cases & $943(46)$ & $47(26-69)$ & $72(40-92)$ \\
\hline
\end{tabular}

mother at birth of the index child (birthweight $r=0.02$; birthweight percentile $r=0.02$ ), the duration of diabetes at birth of the index offspring (birthweight $r=0.01$; birthweight percentile $r=0.03$ ) or the age of the mother at diabetes onset (birthweight $r=0.04$; birthweight percentile $r=0.02)$. Gestational age was correlated with the duration of diabetes in the mother $(r=0.1 ; p=0.001)$ and the age of the mother at diabetes onset $(r=0.12 ; p<0.0001)$.

Intrauterine growth is dependent upon maternal metabolic control In children of mothers with type 1 diabetes, birthweight $(r=0.13 ; p=0.001)$, birthweight percentile $(r=0.26$; $p<0.0001)$ and gestational age $(r=-0.15 ; p=0.0001)$ were significantly associated with maternal $\mathrm{HbA}_{1 c}$ during pregnancy. Women with diabetes are recommended to normalise blood glucose during pregnancy, reflected by $\mathrm{HbA}_{1 c}$ values $<5.6 \%[24,25]$. Birthweight percentiles in children of mothers with type 1 diabetes and $\mathrm{HbA}_{1 c}<5.6 \%$ were close to German reference data and were very high when mothers had an $\mathrm{HbA}_{1 c}>6.5 \%$ (Table 2).

HLA-DR4 identifies maternal diabetes-gene interactions that affect intrauterine growth Consistent with the increased prevalence of DR4 $D Q B 1^{*} 0302$ haplotype in families affected by type 1 diabetes, $48 \%$ of children in our cohorts had DR4 alleles (Table 1). The majority (90\%) of DR4-positive children in this cohort also had the $D Q B 1 * 0302$ allele (not shown). Birthweight percentiles in children of non-diabetic mothers were similar between
DR4-positive and DR4-negative children (Table 2). In contrast, among children born to mothers with type 1 diabetes, birthweight percentiles in DR4-positive children were higher than in their DR4-negative counterparts. The DR4-positive children had a higher birthweight than DR4negative children (mean 3,561 g vs 3,454 g respectively; $p=0.004$ ), whereas gestational age was similar between these two groups (median 38.4 vs 38.3 weeks respectively; $p=0.4$ ). To test whether there was an interaction between maternal type 1 diabetes and child HLA DR4 status on birthweight percentile, both variables and the interaction (maternal type 1 diabetes $\times$ child $H L A-D R 4$ status) were included in a general linear model. The model confirmed that the interaction was significant $(p=0.0004)$.

Birthweight percentile differences between DR4-positive and DR4-negative children were observed in boys $(p=0.001)$ and girls $(p=0.04)$. Differences in birthweight percentile were not observed with regard to HLA DR3 alleles in children of mothers with type 1 diabetes (median 69th birthweight percentile in DR3-positive children vs 69th percentile in DR3-negative children) or in DR4-positive children of mothers with type 1 diabetes (median 77 th percentile in DR3/4-positive children vs 75 th percentile in DR4-positive/DR3-negative children).

We were able to determine the parental origin of HLA DR4 in 441 HLA DR4-positive children, allowing a subanalysis of maternal vs paternal DR4 origin with regard to birthweight percentile (Table 3 ). In children of mothers with type 1 diabetes, birthweight percentile was increased if 
Table 3 Birthweight percentile in relation to parental origin of HLA DR4 in children of type 1 diabetes mothers

\begin{tabular}{lcc}
\hline DR4 alleles in child & $n$ & $\begin{array}{l}\text { Birthweight percentile } \\
\text { (median [IQR]) }\end{array}$ \\
\hline No DR4 allele & 620 & $64(30-90)$ \\
DR4 from father only & 42 & $63(39-89)$ \\
DR4 from mother only & 164 & $75(45-95)^{\mathrm{a}}$ \\
DR4 from both parents & 65 & $82(51-93)^{\mathrm{b}}$ \\
DR4 origin & 270 & $75(42-93)^{\mathrm{a}}$ \\
undetermined & &
\end{tabular}

$\mathrm{IQR}$, interquartile range

${ }^{a} p=0.003$ vs DR4-negative children

${ }^{\mathrm{b}} p=0.004$ vs DR4-negative children

the child inherited the DR4 only from the mother $(p=0.0008)$ or from both mother and father $(p=0.004)$. Again, differences were in birthweight and not gestational age of the child (data not shown). Relatively few children of mothers with type 1 diabetes inherited the DR4 only from the father. These children had similar birthweight percentiles to the non-DR4 children.

Multivariate analysis indicated that birthweight was related to maternal diabetes, gestational age, sex, parity status and HLA DR4 in the child with a total combined $r^{2}$ of $28 \%$ (Table 4). In children of mothers with type 1 diabetes, birthweight was related to gestational age, sex, parity status, HLA DR4 in the child, maternal $\mathrm{HbA}_{1 c}$ during pregnancy and maternal nephropathy, with a total combined $r^{2}$ of $32 \%$. Birthweight percentiles in HLA DR4-positive children born to mothers with type 1 diabetes and a final trimester $\mathrm{HbA}_{1 c}$ $>6.5 \%$ were very high, and in contrast were similar to national reference data in HLA DR4-negative children born to mothers with type 1 diabetes and a final trimester $\mathrm{HbA}_{1 c}$ $<5.6 \%$ (Fig. 1). The relationships between birthweight and maternal diabetes, gestational age, sex, parity status, HLA DR4, nephropathy and $\mathrm{HbA}_{1 c}$ remained significant when only the first participating child from each family was included in the analysis (data not shown).

Birthweight was not significantly different between children with INS VNTR class I/I, I/III or III/III genotypes

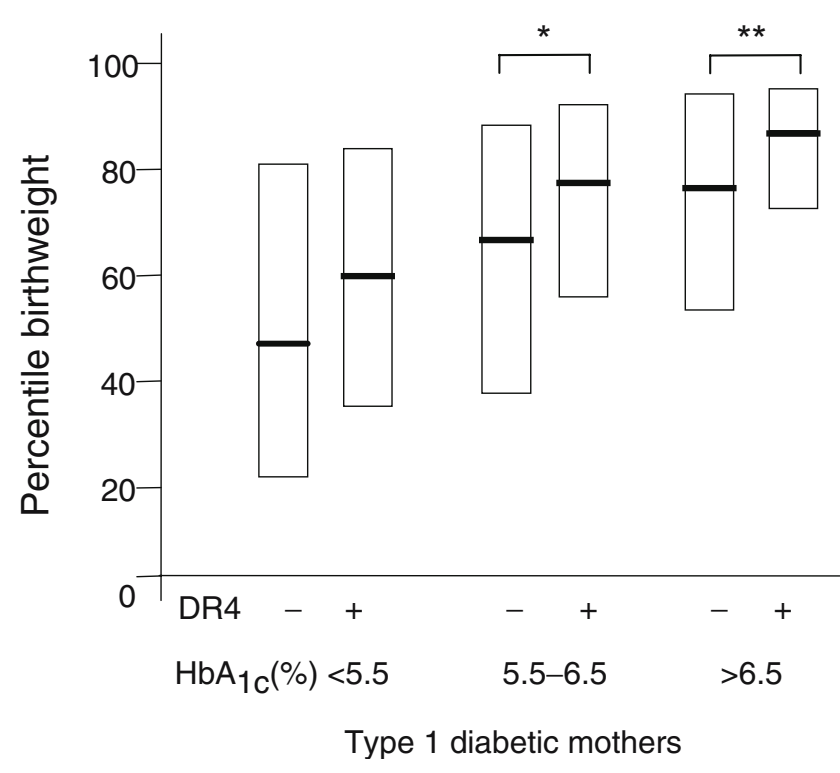

Fig. 1 Birthweight percentiles with regard to maternal $\mathrm{HbA}_{1 c}$ during pregnancy and HLA DR4 status of the child in children of mothers with type 1 diabetes. Birthweight percentiles are birthweights adjusted for gestational age and sex derived from national reference data [19]. Boxes represent the median and interquartile range. $* p<0.05 ; * * p<0.01$. Numbers of type 1 diabetic mothers for DR4- and DR4+, respectively: $\mathrm{HbA}_{1 c}<5.5 \%, n=135, n=112 ; \mathrm{HbA}_{1 c}$ 5.5-6.5\%, $n=132, n=135$; $\mathrm{HbA}_{1 c}>6.5 \%, n=48, n=44$

(Table 2). No differences with regard to INS VNTR genotypes were observed when only children of mothers with type 1 diabetes were examined. INS VNTR genotypes were in Hardy-Weinberg equilibrium (chi-squared, $0.0016)$.

Weight, height and BMI during infancy Weight and BMI percentiles at age 2 and 5 years in relation to maternal diabetes, sex, birthweight and DR4 status of the child are provided in the Electronic supplementary material (ESM) Table 1. Birthweight percentile was the strongest predictor of weight and BMI percentiles at age 2 and 5 years (Fig. 2, ESM Table 1). Weight percentile and BMI percentile at age 5 years were correlated with birthweight (weight percentile $r=0.25 ; p<0.0001$; BMI percentile $r=0.2 ; p<0.0001$ ), but not with gestational age ( $r=0.03$ and $r=0.01$ respectively).
Table 4 Multivariate analysis of factors associated with birthweight

${ }^{\text {a }}$ Adjusted for maternal age and duration of diabetes.

\begin{tabular}{|c|c|c|c|c|}
\hline \multirow[t]{2}{*}{ Variable } & \multicolumn{2}{|l|}{ All children } & \multicolumn{2}{|c|}{ Children of mothers with type 1 diabetes } \\
\hline & Beta coefficient & $p$ value & Beta coefficient ${ }^{\mathrm{a}}$ & $p$ value $^{\mathrm{a}}$ \\
\hline Maternal type 1 diabetes & 0.22 & $<10^{-10}$ & - & - \\
\hline Gestational age (weeks) & 0.51 & $<10^{-10}$ & 0.47 & $<10^{-10}$ \\
\hline Boys & 0.11 & $<10^{-9}$ & 0.11 & 0.0009 \\
\hline Parity status & 0.16 & $<10^{-10}$ & 0.18 & $<10^{-6}$ \\
\hline DR4 & 0.05 & 0.006 & 0.09 & 0.006 \\
\hline Maternal $\mathrm{HbA}_{1 c}(\%)$ & - & - & 0.16 & $<10^{-6}$ \\
\hline Overt nephropathy & - & - & -0.15 & $<10^{-5}$ \\
\hline
\end{tabular}


a

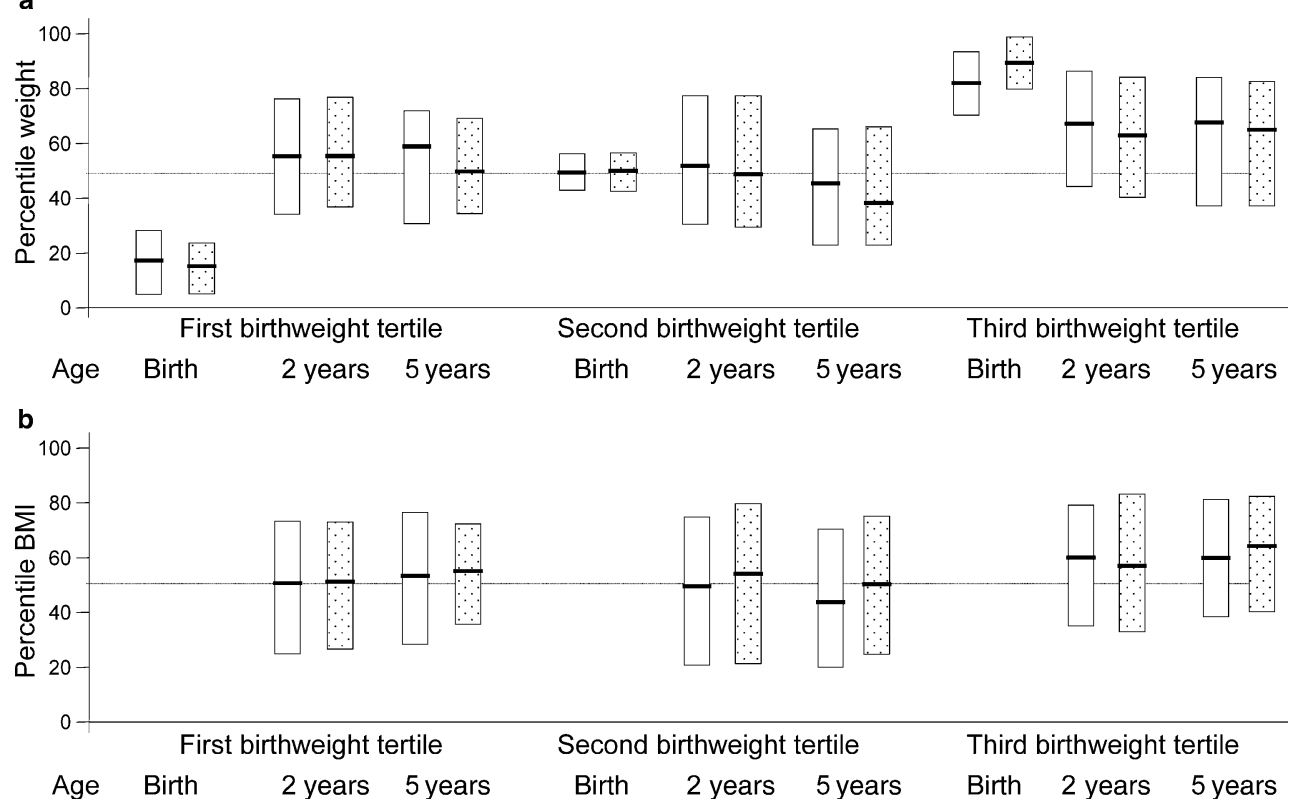

Fig. 2 Weight (a) and BMI (b) during childhood in relation to birthweight and maternal diabetes. Children are classified according to their birthweight (first, second and third tertiles after adjusting for gestational age and sex relative to 563,480 German control offspring [20]), and according to the type 1 diabetes status of their mother (open boxes, non-diabetic mothers; dotted boxes, mothers with type 1 diabetes). Weight and BMI at age 2 years and at age 5 years are

Similar relationships were also observed for weight percentile and BMI percentile at age 2 years (not shown). Factors associated with increased birthweight (maternal diabetes, DR4 status of child in mothers with type 1 diabetes) were marginally and inconsistently related to weight and BMI percentiles in childhood. INS VNTR genotype in the total cohort or in children of mothers with type 1 diabetes were not associated with weight and BMI at ages 2 and 5 years (not shown).

By multivariate analysis, birthweight $\left(p<10^{-10}\right)$ and gestational age $(p=0.001)$, but not maternal diabetes $(p=0.5)$ or DR4 status in the child $(p=0.9)$ were predictors of BMI percentile at ages 2 and 5 years. Birthweight $\left(p<10^{-10}\right)$ and gestational age $(p=0.004)$ were predictors of weight percentile at ages 2 and 5 years. Multivariate analysis in children of mothers with type 1 diabetes indicated that birthweight and gestational age are predictors of weight and BMI percentiles, but did not find evidence of an effect by HLA DR4 status of the child on weight percentile $(p=0.4)$ and BMI percentile $(p=0.9)$ at ages 2 and 5 years.

The dominant relationship of birthweight to weight and BMI in childhood and the marked increase in birthweight in children of mothers with type 1 diabetes meant that children of mothers with type 1 diabetes were more likely to be overweight (BMI >90th percentile of normative expressed as percentiles adjusted for age and sex from national reference data [20]. Boxes represent the median and interquartile range. Numbers of children per column, from left to right: first tertile: $n=303$, $n=267, n=202, n=222, n=137, n=166$; second tertile: $n=295, n=279$, $n=227, n=223, n=158, n=169$; third tertile: $n=274, n=615, n=203, n=$ $485, n=129, n=359$

population) at age 5 years than children from non-diabetic mothers (13.4 vs $8 \% ; p=0.006)$. Mothers with type 1 diabetes who had $\mathrm{HbA}_{1 c}>6.5 \%$ during pregnancy had the highest proportion of overweight children $(23 \%$ at age 5 years).

\section{Discussion}

Maternal diabetes was found to influence intrauterine growth in an interactive manner both with metabolic control during pregnancy and with presence in the child of the type 1 diabetes susceptibility HLA DR4 alleles. Birthweight was a determinant of weight during childhood, regardless of maternal diabetes.

The study was performed on the largest cohort of prospectively followed newborn children with first-degree relatives who have type 1 diabetes, and in particular of children born to mothers with type 1 diabetes. Children were recruited throughout Germany. Weight and height data were obtained from obstetric and paediatric records, and all weight, height and BMI data were related to national reference birth data from more than two-million singleton births in the 1990s [20] (M. Voigt, unpublished data] and to childhood registry data. Validation of our data showed that children of non-diabetic mothers in our cohort had adjusted 
birthweights similar to those of the reference cohort and that their median weight and BMI during childhood were at or close to the 50th percentile of that cohort. Nevertheless, the study cohort was not population-based, and participation may have been biased in favour of better educated families or families fluent in the German language.

Another potential limitation of the study is that maternal $\mathrm{HbA}_{1 c}$ and INS VNTR genotyping were not obtained in all cases. Although birthweight percentiles in the missing cases appeared to be representative of the remainder of the cohort, we cannot exclude the possibility that the findings might have been different if these data had been obtained. Moreover, $\mathrm{HbA}_{1 c}$ measurements were performed locally using different assays and the relationship between maternal $\mathrm{HbA}_{1 c}$ and birthweight might have differed, if $\mathrm{HbA}_{1 c}$ had been measured centrally with a single method. Finally, there was co-linearity between some of the variables analysed, and some variables are likely to be influenced by other confounder variables, such as socio-ecomonic status, education, maternal BMI and hypertension during pregnancy, which were not available for the analysis. Thus, observed associations could in some cases be secondary to other unknown variables.

Maternal diabetes had a marked effect on birthweight and gestational age. As previously reported [26], this effect was partially related to metabolic control during pregnancy. Our data and previous data on much fewer subjects [27] indicate that metabolic control as measured by $\mathrm{HbA}_{1 c}$ during the final trimester is an important contributor to the increased birthweight in children of mothers with diabetes. The mechanism of this phenomenon is not completely understood, but elevated levels of fetal insulin, leptin and lipids are potentially responsible [28]. Other factors are likely to be involved. In particular, genetic factors are thought to influence fetal growth [12, 29-32].

An intriguing observation was recently reported in children born in southern Sweden, according to which children with the type 1 diabetes susceptibility haplotype HLA DR4_DQB1*0302 had increased birthweight [12]. The study was performed in more than 20,000 unselected births, including more than 1,500 children who had at least one type 1 diabetes susceptible HLA $D Q B 1^{*} 0302$ haplotype. We were unable to detect DR4-related differences in the 872 children born to non-diabetic mothers in our study. However, we were unlikely to have the statistical power to formally test the relatively small differences observed in the Swedish study. We were also unable to confirm earlier findings from 979 births in Norway [13] of increased birthweights in children with the type 1 diabetes-protective HLA $D Q B 1^{*} 0602$ haplotypes (data not shown). Again, numbers of children with the HLA $D Q B 1 * 0602$ haplotypes were relative low in our cohort (14\% of cohort). Remarkably, and in support of the findings in Sweden, we did find differences between birthweights in DR4positive and -negative children born to mothers with type 1 diabetes, suggesting that maternal diabetes amplifies the relationship between HLA DR4 alleles and birthweight. A sub-analysis of the parental origin of the DR4 showed that birthweight was increased if children inherited HLA DR4 from mothers who also had type 1 diabetes. Although no increase in birthweight was seen if the child inherited HLA DR4 from the father, the study had insufficient power to exclude an effect by the paternal DR4. Similarly, the study had insufficient numbers of children who were HLA DR4 homozygous to determine whether birthweight was further increased in these children. Moreover, because most DR4-positive children had the HLA DQB1*0302 allele, it was not possible to determine whether HLA DR or DQ was associated with birthweight. It is, of course, possible that not all DR4 subtypes are associated with increased birthweight. We therefore conclude that the presence of HLA DR4, either in the child, or in the mother, is indirectly involved in intrauterine growth or marks other loci that affect intrauterine growth. How HLA DR or other loci affect fetal growth is unclear. In our study, both HLA DR4 and maternal $\mathrm{HbA}_{1 c}$ contributed to the increased birthweight in children of mothers with type 1 diabetes. Furthermore, HLA DR4-positive children had increased weight, even if diabetic mothers had normal $\mathrm{HbA}_{1 c}$ during pregnancy, suggesting that the effect of HLA DR was not strictly related to metabolic control, but that other factors such as insulinaemia could contribute to the HLA DR4 effect on birthweight.

The INS VNTR type 1 diabetes susceptibility locus has been inconsistently reported to affect birthweight and growth during childhood [29, 30, 33-35]. Homozygosity for INS VNTR class III alleles was associated with increased birthweight in a subgroup of children from south-west England who did not show 'catch-up' or 'catch-down' weight changes from birth to age 2 years [29]. The INS VNTR class III allele was associated with reduced birthweight in Pima Indians [30], whereas studies in much larger cohorts from England, Finland and Norway failed to find any association between INS VNTR genotypes and birthweight [33-35]. Similarly, an association between INS VNTR genotypes and weight in childhood and adulthood was also reported, but a more recent report failed to confirm the original findings [36]. We did not find a significant difference in birthweight and weight or BMI during childhood between children who had INS VNTR class I/I and class I/III genotypes. This applied even when the analysis was restricted to children of mothers with type 1 diabetes, i.e. children who are likely to have been exposed to higher concentrations of insulin during fetal growth. No significant differences in birthweight, gestational age or birthweight percentile were seen in children 
who had the class III/III genotype as compared with children with class I/I, but the number of children with the class III/III genotype was low in our cohort.

Birthweight was a determinant of weight and BMI during childhood. Compared with children with birthweights in the lowest tertile of the normative population, children who had birthweights in the upper tertile of the reference normative population were twice as likely to have high weight and BMI and be classified as overweight ( $>90$ th centile of normative population) at age 5 years. This was true both for children of non-diabetic mothers and for those of mothers with type 1 diabetes. Accordingly, since children of mothers with type 1 diabetes had high birthweights, they were also more likely to be overweight at age 5 years than children of non-diabetic mothers. Mothers with type 1 diabetes who had high $\mathrm{HbA}_{1 c}(>6.5 \%)$ during pregnancy had the highest proportion of overweight children at age 5 years. Thus, children of mothers with diabetes and children with high birthweight could be potential targets for programmes aimed at reducing childhood obesity.

The associations between maternal diabetes, high birthweight and weight gain during childhood are potentially relevant to risk of childhood type 1 diabetes. The associations, however, are somewhat contrary to expectations. Although the effects are relatively weak, high birthweight and increased childhood growth rates are postulated to increase the risk of type 1 diabetes [37-39]. Yet, despite their high birthweight and increased BMI during childhood, children of mothers with type 1 diabetes have a lower risk of type 1 diabetes than children of fathers with type 1 diabetes [40]. Thus, there are likely to be important maternal factors that reduce the risk of type 1 diabetes and nullify other risk factors. The identification of these factors could help reduce the incidence of type 1 diabetes.

Acknowledgements This study was supported by grants from the German Research Foundation (Deutsche Forschungsgemeinschaft ZI 310/14-1 and 14-2), the Juvenile Diabetes Research Foundation (JDRF no. 1-2000-619) and the Foundation 'Children with type 1 diabetes' (Stiftung 'Das Zuckerkranke Kind'). We thank A. Baumgarten, C. Winkler and A. Locher for expert technical assistance and all families and paeditricians in Germany for participating in the study.

Duality of interest None of the authors involved in this study had any duality of interest.

\section{References}

1. Daniels SR, Arnett DK, Eckel RH et al (2005) Overweight in children and adolescents: pathophysiology, consequences, prevention, and treatment. Circulation 111:1999-2012
2. Paterson CC, Dahlquist G, Soltesz G et al (2001) Is childhoodonset type I diabetes a wealth-related disease? An ecological analysis of European incidence rates. Diabetologia 44 (Suppl 3): B9-B16

3. St-Onge MP, Keller KL, Heymsfield SB (2003) Changes in childhood food consumption patterns: a cause for concern in light of increasing body weights. Am J Clin Nutr 78:1068-1073

4. Agras WS, Mascola AJ (2005) Risk factors for childhood overweight. Curr Opin Pediatr 17:648-652

5. Gillman MW, Rifas-Shiman S, Berkey CS et al (2003) Maternal gestational diabetes, birth weight, and adolescent obesity. Pediatrics 111:221-226

6. Rogers I, EURO-BLCS Study Group (2003) The influence of birthweight and intrauterine environment on adiposity and fat distribution in later life. Int J Obes Relat Metab Disord 27:755777

7. Parsons TJ, Power C, Logan S et al (1999) Childhood predictors of adult obesity: a systematic review. Int J Obes Relat Metab Disord 23:S1-S107

8. Ong KK, Dunger DB (2004) Birth weight, infant growth and insulin resistance. Eur J Endocrinol 151(Suppl 3):U131-U139

9. Williams LA, Evans SF, Newnham JP (1997) Prospective cohort study of factors influencing the relative weights of the placenta and the newborn infant. BMJ 314:1864-1868

10. Silverman BL, Rizzo T, Cho NH et al (1991) Long-term prospective evaluation of offspring of diabetic mothers. Diabetes 40:121-125

11. Hod M, Merlob P, Friedman S et al (1991) Gestational diabetes mellitus. A survey of perinatal complications in the 1980s. Diabetes 40(Suppl 2):74-78

12. Larsson HE, Lynch K, Lernmark B et al (2005) Diabetesassociated HLA genotypes affect birthweight in the general population. Diabetologia 48:1484-1491

13. Stene LC, Magnus P, Ronningen KS, Joner G (2001) Diabetesassociated HLA-DQ genes and birth weight. Diabetes 50:28792882

14. Wilkin TJ (2001) The accelerator hypothesis: weight gain as the missing link between Type 1 and Type 2 diabetes. Diabetologia 44:914-922

15. Dahlquist G (2006) Can we slow the rising incidence of childhood-onset autoimmune diabetes? The overload hypothesis. Diabetologia 49:20-24

16. Hummel M, Bonifacio E, Schmid S et al (2004) Islet autoantibody development and risk for childhood Type 1 diabetes in offspring of affected parents. Ann Intern Med 140:882-886

17. Ziegler AG, Hillebrand B, Rabl W et al (1993) On the appearance of islet associated autoimmunity in offspring of diabetic mothers: a prospective study from birth. Diabetologia 36:402-408

18. Ziegler AG, Hummel M, Schenker M et al (1999) Autoantibody appearance and risk for the development of childhood diabetes in offspring of parents with Type 1 Diabetes: The German BABYDIAB Study. Diabetes 48:460-468

19. Schmid S, Buuck D, Knopff A et al (2004) BABYDIET, a feasibility study to prevent the appearance of islet autoantibodies in relatives of patients with Type 1 diabetes by delaying exposure to gluten. Diabetologia 47:1130-1131

20. Voigt M, Schneider KTM, Jahrig K (1996) Analysis of a 1992 birth sample in Germany. New percentile values of the body weight of newborn infants. Geburtshilfe Frauenheilkd 56:550-558 [Article in German]

21. Walter M, Albert E, Conrad M et al (2003) IDDM2/insulin VNTR modifies risk conferred by IDDM1/HLA for development of type 1 diabetes and associated autoimmunity. Diabetologia 46:712-720

22. Kimura A, Sasazuki T (1992) 11th International Histocompatibility Workshop reference protocol for the HLA DNA-typing 
technique. In: Tsuji K, Aizawa A, Sasazuki T (eds) HLA. Vol. 1, Oxford University Press, Oxford pp 397-419

23. Bennett ST, Lucassen AM, Gough SC et al (1995) Susceptibility to human type 1 diabetes at IDDM2 is determined by tandem repeat variation at the insulin gene minisatellite locus. Nat Genet 9:284-292

24. Nielsen LR, Ekbom P, Damm P et al (2004) HbAlc levels are significantly lower in early and late pregnancy. Diabetes Care 27:1200-1201

25. ADA (2004) Clinical practice recommendations. gestational diabetes mellitus. Diabetes Care 27:S88-S90

26. Mello G, Parretti E, Mecacci F et al (2000) What degree of maternal metabolic control in woman with type 1 diabetes is associated with normal body size and proportions in full-term infants? Diabetes Care 23:1494-1498

27. Johnstone FD, Mao JH, Steel JM et al (2000) Factors affecting foetal weight distribution in women with type 1 diabetes. BJOG 107:1001-1006

28. Lepercq J, Taupin P, Dubois-Laforgue D et al (2001) Heterogeneity of foetal growth in type 1 diabetic pregnancy. Diabetes Metab 27:339-344

29. Dunger DB, Ong KK, Huxtable SJ et al (1998) Association of the INS VNTR with size at birth. ALSPAC study team. Avon longitudinal study of pregnancy and childhood. Nat Genet 19:98-100

30. Lindsay RS, Hanson RL, Wiedrich C et al (2003) The insulin gene variable number tandem repeat class I/III polymorphism is in linkage disequilibrium with birth weight but not type 2 diabetes in the Pima population. Diabetes 52:187-193

31. Dunger DB, Petry CJ, Ong KK (2006) Genetic variations and normal fetal growth. Horm Res 65(Suppl 3):34-40

32. Fradin D, Boileau P, Lepercq J, Bougneres P (2006) 'Non-Mendelian' genetics of fetal growth. J Endocrinol Invest 29(Suppl 1):11-15
33. Mitchell SM, Hattersley AT, Knight B et al (2004) Lack of support for a role of the insulin gene variable number of tandem repeats minisatellite (INS-VNTR) local in foetal growth or type 2 diabetes-related intermediate traits in United Kingdom populations. J Clin Endocrinol Metab 89:310-317

34. Bennett AJ, Sovio U, Ruokonen A et al (2004) Variation at the insulin gene polymorphism VNTR (Variable number tandem repeat) polymorphism and early growth. Diabetes 53:21262131

35. Stene LC, Thorsby PM, Berg JP et al (2006) The relation between size at birth and risk of type 1 diabetes is not influenced by adjustment for the insulin gene $(-23 \mathrm{HphI})$ polymorphism or HLA-DQ genotype. Diabetologia 49:2068-2073

36. Ong KK, Petry CJ, Barratt BJ et al (2004) Maternal-foetal interactions and birth order influence insulin variable number of tandem repeats allele class associations with head size at birth and childhood weight gain. Diabetes 53:1128-1133

37. Dahlquist G, Bennich SS, Källén B (1996) Intrauterine growth pattern and risk of childhood onset insulin dependent (type 1) diabetes: population based case-control study. BMJ 313:11741177

38. Stene LC, Magnus P, Lie RT et al (2001) Birth weight and childhood onset type 1 diabetes: population based cohort study. BMJ 322:889-892

39. Dahlquist GG, Pundziute-Lycka A, Nystrom L; Swedish Childhood Diabetes Study Group; Diabetes Incidence Study in Sweden (DISS) Group (2005) Birthweight and risk of type 1 diabetes in children and young adults: a population-based register study. Diabetologia 48:1114-1147

40. Warram JH, Krolewski AS, Gottlieb MS et al (1984) Differences in risk of insulin-dependent diabetes in offspring of diabetic mothers and diabetic fathers. N Engl J Med 311:149-152 